\title{
In vitro development of mouse fetal germ cells into mature oocytes
}

\author{
Wei Shen ${ }^{1,2}$, Lan $\mathrm{Li}^{1}$, Zhaodai Bai ${ }^{2,3}$, Qingjie Pan ${ }^{1}$, Mingxiao Ding ${ }^{2}$ and Hongkui Deng ${ }^{2}$ \\ ${ }^{1}$ College of Animal Science and Technology, Institute of Animal Reproduction Development and Genetic Engineering, \\ Qingdao Agricultural University, Qingdao 266109, China, ${ }^{2}$ College of Life Sciences, Peking University, Beijing \\ 100071, China and ${ }^{3}$ Department of Female Clinic Research, National Research Institute for Family Planning, Beijing \\ 100071, China
}

Correspondence should be addressed to W Shen; Email: shenwei427@yahoo.com.cn

\begin{abstract}
Little is known about the mechanisms underlying primordial follicular formation and the acquisition of competence to resume meiosis by growing oocytes. It is therefore important to establish an in vitro experimental model that allows one to study such mechanisms. Mouse follicular development has been studied in vitro over the past several years; however, no evidence has been presented showing that mature oocytes can be obtained from mouse fetal germ cells prior to the formation of primordial follicles. In this study, a method has been established to obtain mature oocytes from the mouse fetal germ cells at 16.5 days postcoitum (dpc). From the initiation of primordial follicular formation to the growth of early secondary follicles, ovarian tissues from 16.5 dpc fetal mice were cultured in vitro for 14 days. Subsequently, 678 intact secondary follicles were isolated from 182 mouse fetal ovaries and cultured for 12 days. A total of 141 oocytes inside antral follicles were matured in vitro, and 102 oocytes underwent germinal vesicle breakdown. We found that 97 oocytes were fertilized and 15 embryos were able to form morula-blastocysts. We also analyzed various genomic imprinting markers and showed that the erasure of genomic imprinting markers in the parental generation was also imposed on the oocytes that developed from fetal germ cells. Our results demonstrate that mouse fetal germ cells are able to form primordial follicles with ovarian cells, and that oocytes within the growing follicles are able to mature normally in vitro.
\end{abstract}

Reproduction (2007) 134 223-231

\section{Introduction}

The assembly and subsequent development of primordial follicles are crucial for mouse oogenesis. Primordial follicles are formed from granulosa cells encasing individual oocytes in the mouse fetal ovary after 17 days of gestation (Pepling \& Spradling 1998, 2001, Epifano \& Dean 2002). Female germ cells within the mouse fetal ovary are arrested in the prophase of the first meiotic division, and become surrounded by a single layer of granulosa cells contained within an outer basal lamina (Byskov et al. 1997, Kezele et al. 2005). Interactions between oocytes and pregranulosa cells are crucial for the initial formation of primordial follicles, and the initial stages of folliculogenesis are independent of gonadotropins and involve cell-autonomous and non-cellautonomous factors (Epifano \& Dean 2002). With periodicity, cohorts of these primordial follicles, representing the lifetime complement of germ cells available to females, are recruited into a growth phase that culminates in meiotic maturation and ovulation of the mature oocytes into the oviduct in preparation for fertilization (Epifano \& Dean 2002). Regulative mechanisms of primordial follicle formation and development are only partially understood, and more information underlying these processes could be obtained by culturing follicles in vitro at the earliest stages of formation and growth (Smitz \& Cortvrindt 2002).

During the last few years, the formation and development of mouse primordial follicles have been investigated in vitro (Eppig \& O'Brien 1996, Eppig et al. 1996, Klinger \& de Felici 2002, Obata et al. 2002a, O'Brien et al. 2003, Lenie et al. 2004, Niwa et al. 2004, Shen et al. 2006a, 2006b). The primordial follicles within the newborn mouse ovaries were able to achieve complete development and maturation in vitro, although the frequency of preimplantation development was low (Eppig \& O'Brien 1996, Eppig et al. 1996, O'Brien et al. 2003). The mature oocytes could not be directly obtained from the mouse fetal germ cells using in vitro culture (McLaren \& Southee 1997, McLaren 2000, Chuma \& Nakatsuji 2001, Klinger \& de Felici 2002, 
Obata et al. 2002a; Niwa et al. 2004). Mouse oocytes could develop to the size of $50 \mu \mathrm{m}$ from 16.5 days postcoitum (dpc) fetal germ cells and show signs of chromatin differentiation, but the oocytes were unable to go on to acquire meiotic/developmental competence in vitro (Klinger \& de Felici 2002). However, several studies have demonstrated that the developmental potential of mouse primordial follicles could be restored by the transplantation of ovaries under the kidney capsule (Cox et al. 2000, Liu et al. 2000, 2001). Recently, using the ectopic transplantation technology, our group demonstrated that oocytes generated from the $16.5 \mathrm{dpc}$ fetal germ cells had the potential to be fertilized in vitro and to support early embryonic development (Shen et al. 2006a). However, regulation of the in vitro formation of mouse primordial follicle derived from fetal germ cells, the selection and initiation of growth of the primordial follicles out of the dormant pool, and the oocytes generated in vitro that had full developmental potential to support the embryonic development remain enigmatic. As the store of female gametes is maintained within the primordial follicles, the real challenge is to establish an in vitro culture system for studying primordial follicles. The formation and development of primordial follicles, as well as the transition from primordial to mature follicle, can be studied using this system. Finally, this system could potentially provide an almost unlimited source of oocytes for biomedical use (Smitz \& Cortvrindt 2002).

The purpose of this study is to determine the developmental competence of the germ cells from the $16.5 \mathrm{dpc}$ fetal ovaries to differentiate into mature oocytes by in vitro culture. The results demonstrate that $16.5 \mathrm{dpc}$ mouse fetal germ cells are able to aggregate into primordial follicles with the ovarian pregranulosa cells in vitro, and the oocytes from the growing follicles are able to mature and support early embryonic development in vitro.

\section{Material and Methods}

\section{Ovarian tissue isolation and culture in vitro}

Upon the approval of study plan by the Institutional Animal Care and Use Committees of Qingdao Agricultural University, $16.5 \mathrm{dpc}$ mouse fetal ovaries were obtained from pregnant CD-1 mice (Vital River, Beijing, China), and the copulation plug was designated at 0.5 dpc. The $16.5 \mathrm{dpc}$ mouse fetal ovaries without attached mesonephroses were cultured in vitro. Briefly, the ovaries were placed on Millicell-PC membrane inserts (3.0 $\mu \mathrm{m}$ pore size, $10 \mathrm{~mm}$ diameter; Millipore Corp., Medford, MA, USA) with medium filling only the lower chamber, and each ovary was placed on each membrane. Then the medium was removed from the lower chamber until only a thin film covered the ovaries. The medium for organ culture was DMEM/F12+ + -MEM
(1:1; Gibco-BRL) supplemented with $0.23 \mathrm{mM}$ pyruvic acid, 3\% (w/v) BSA (Sigma), insulin-transferrinselenium mix (ITS-mix; $5 \mathrm{mg} / \mathrm{ml}, 5 \mathrm{mg} / \mathrm{ml}$, and $5 \mathrm{ng} / \mathrm{ml}$ respectively; Sigma), $100 \mathrm{U} / \mathrm{ml}$ penicillin $\mathrm{G}$, and $100 \mathrm{mg} / \mathrm{ml}$ streptomycin sulfate. The organ cultures were maintained for 14 days at $37^{\circ} \mathrm{C}$ in modular incubation chambers (Billups Rothenberg, Del Mar, CA, USA) thoroughly infused with a gas mixture of $5 \%$ $\mathrm{CO}_{2}$ and $95 \%$ air (O'Brien et al. 2003). The medium was changed every $48 \mathrm{~h}$ with replacement of half of the complete medium.

\section{Follicles isolation and culture in vitro}

Fourteen days after mouse ovarian tissue culture, the intact early secondary follicles $(110-130 \mu \mathrm{m})$ were mechanically isolated from the cultured ovaries using needles, and then cultured in vitro for 12 days (Liu et al. 2001, Shen et al. 2006b). Selected follicles were individually washed and placed in the culture droplets. Medium A consisted of $\alpha$-MEM with $5 \%$ heat-inactivated fetal calf serum (Gibco-BRL), $100 \mathrm{mlU} / \mathrm{ml}$ folliclestimulating hormone $(\mathrm{FSH}), 10 \mathrm{mlU} / \mathrm{ml}$ luteinizing hormone (LH; Sigma), and ITS-mix. Culture dishes (10 cm Petri dish; BD Bioscience, Bedford, MA, USA) containing sixty $10 \mu \mathrm{l}$ culture droplets covered with $10 \mathrm{ml}$ mineral oil were preincubated overnight at $37^{\circ} \mathrm{C}$, $100 \%$ humidity, and $5 \% \mathrm{CO}_{2}$ in the air. On day 2 after culture, $10 \mu \mathrm{l}$ fresh medium A were added to each droplet. From day 4 onward, the depleted medium was refreshed by exchanging $10 \mu \mathrm{l}$ fresh medium every other day. The diameters of mouse follicles and oocytes cultured in vitro were measured daily. On day 12, $10 \mu \mathrm{l}$ medium were replaced with $10 \mu \mathrm{l}$ mature medium $B$ to induce final oocyte maturation. Mature medium B consisted of $\alpha$-MEM supplemented with $1.5 \mathrm{IU} / \mathrm{ml}$ rhCG (Sigma) and $5 \mathrm{ng} / \mathrm{ml}$ recombinant epidermal growth factor (rEGF; Sigma). After 16-18 h, cumulus expansion of the cumulus-oocyte complexes (COCs) was assessed. Oocyte maturation status was adjudged by germinal vesicle breakdown (GVBD) and extrusion of the first polar body (Shen et al. 2006a, 2006b).

The early secondary follicles were mechanically isolated from the 12-day-old CD-1 ovaries in the control group, and were cultured in vitro for 12 days using the protocol as described above for in vitro growth.

\section{Assessment of steroid production in vitro}

From follicle culture of day 6 , medium A was refreshed every 2 days by retrieving and adding $10 \mu \mathrm{l}$. From the surviving follicles, the medium was pooled per culture dish and stored at $-20^{\circ} \mathrm{C}$ for hormone analysis. Estradiol $\left(E_{2}\right)$ and progesterone were measured using RIAs; $E_{2}$ was measured using a very sensitive $(4 \mathrm{pg} / \mathrm{l})$ and reproducible RIA (total coefficient of variation (CV), 10\%) from clinical 
assays with a measurement range for $E_{2}$ from 4 to $500 \mathrm{ng} / \mathrm{l}$. Progesterone secretion was measured using RIA from CIS Biointernational with an analytical sensitivity of $40 \mathrm{ng} / \mathrm{l}$ and a precision of $8 \%$ or less (CV \%). The assays of $E_{2}$ were validated for use by sampling the culture medium every other day, and the assays of progesterone were validated at days 12 and 13 respectively (Lenie et al. 2004, Shen et al. 2006a).

\section{Expression of specific genes in the different follicular stages}

RNA samples were isolated from the different stages of follicles $(n=10)$, and oligo-dT-primed cDNA was synthesized using superscript reverse transcriptase (RT) according to the manufacturer's protocol (TaKaRa, Kyoto, Japan). One microliter of each RT reaction product was used in each $25 \mu \mathrm{l}$ PCR mix primed with gene-specific oligonucleotides. The following primers were used to detect mouse-specific genes in the different follicular stages: $D d x 4$ is a marker for postmigratory germ cells until postmeiotic stages, 5'-GGT CCA AAA GTG ACA TAT ATA CCC-3' and 5'-TTG GTT GAT CAG TTC TCG AGT-3' (420 bp long); growth differentiation factor 9 (Gdf9), 5'-CCA GCA GAA GTC ACC TCT ACA A$3^{\prime}$ and 5'-ACA TGG CCT CCT TTA CCA CA-3' (240 bp long); the mouse homolog of the yeast meiosis-specific homologous recombination gene, disrupted meiotic cDNA 1 homolog $(D m c 1 h)$, 5'-GGA CAT TGC TGA CCG CTT CAA CGT- $3^{\prime}$ and $5^{\prime}$-GGC GAT CCT CAG TTC TCC TCT TCC-3' (427 bp long); Og2x is an oocytespecific homeobox gene expressed in germ cell cysts and in primordial and growing oocytes, 5'-CCT TCA GTC ACA GTT TCC GTA T-3' and 5'-GGG AGG TTC TGG CAA GCA AT-3' (226 bp long); the synaptonemal complex protein 3 (Sycp3), 5'-ATG ATG GAA ACT CAG CAG CAA GAG A-3' and 5'-TTG ACA CAA TCG TGG AGA GAA CAA C-3' (325 bp long); and LH/choriogonadotropin receptor ( hcgr), 5'-AAT CTC TCC TTT GCA GAC TTT TG-3' and 5'-AGC ATA GGT GAT GGT GTG CCA-3' (216 bp long). Gapdh primers were used as a control supplied by the RT-PCR kit. The cycling conditions were as follows: $30 \mathrm{~s}$ at $94{ }^{\circ} \mathrm{C}, 30 \mathrm{~s}$ at $60{ }^{\circ} \mathrm{C}$, and $1 \mathrm{~min}$ at $72{ }^{\circ} \mathrm{C}$ (25 cycles; Shen et al. 2006b).

\section{DNA methylation analysis}

Genomic DNA was prepared from the oocytes $(n=15)$ that developed in vitro from mouse fetal germ cells in experimental group, control group, and normal mice. DNA was treated with a sodium bisulfite solution as described previously (Shen et al. 2006b). Region 2 of the Igf $2 r$ gene and the $5^{\prime}$ upstream region of Peg3 were amplified using Ex-Taq DNA polymerase (TaKaRa). The PCR primers were as follows: Igf2r, 5'-GAG GTT AAG GGT GAA AAG TTG TAT- $3^{\prime}$ and $5^{\prime}$-CAC TTT TAA ACT
TAC CTC TCT TAC-3' (450 bp long); Peg3, 5'-TTT TGT AGA GGA TTT TGA TAA GGA GGT G-3' and $5^{\prime}$-CCC CAA ACA CCA TCT AAA CTC TAC AAA C-3' (288 bp long). The cycling conditions were as follows: $30 \mathrm{~s}$ at $94{ }^{\circ} \mathrm{C}, 30 \mathrm{~s}$ at $60^{\circ} \mathrm{C}$, and $1 \mathrm{~min}$ at $72^{\circ} \mathrm{C}$ (35 cycles). Amplified fragments were cloned into the plasmid vector pGEM-T Easy (Promega), and 20 samples in each experiment sequenced using an ABI PRISM 3100 Genetic Analyzer (Applied Biosystems, Foster City, CA, USA).

\section{In vitro fertilization and embryonic culture in vitro}

To test the developmental competence of the oocytes derived from fetal germ cells in vitro, the oocytes were fertilized in vitro. The caudal epididymis was removed from 10- to 12-week-old CD-1 male mice and placed in $1 \mathrm{ml}$ drops of a mutant potassium simplex optimized medium (mKSOM) supplemented with $0.4 \%(\mathrm{w} / \mathrm{v}) \mathrm{BSA}$ in sperm dispersion dishes. The dispersion dishes were placed in a $5 \% \mathrm{CO}_{2}$ incubator for $20 \mathrm{~min}$ to allow the sperm to disperse. Sperm suspension of $10 \mu$ I was added to $90 \mu \mathrm{l}$ drops of mKSOM+BSA in the fertilization dishes. Capacitation was allowed to proceed for 45-60 min at $37^{\circ} \mathrm{C}$ in the incubator. After capacitation, COCs were transferred to the $100 \mu$ fertilization droplets. Incubation was allowed to proceed for $4 \mathrm{~h}$ at $37{ }^{\circ} \mathrm{C}$ in a $5 \% \mathrm{CO}_{2}$ atmosphere. Finally, about ten inseminated oocytes were incubated in $20 \mu \mathrm{l}$ drops of $\mathrm{KSOM}+\mathrm{BSA}$ in culture dishes for $48 \mathrm{~h}$. Then the medium was replaced with $20 \mu \mathrm{l}$ mKSOM+BSA, continuing culture for $48 \mathrm{~h}$. On day 4 , the embryonic development was determined at the late morula-early blastocyst stages (Shen et al. 2006a, 2006b).

\section{Statistical methods}

Follicle formation and survival data, oocyte and follicle diameter, and nuclear maturity were analyzed using $\chi^{2}$-analysis for likely trends. The oocyte proportions that underwent GVBD, fertilization, and late morula-early blastocyst formation were compared by contingency table analysis, followed by $\chi^{2}$-testing. Probability $<0.05$ was considered statistically significant.

\section{Results}

\section{Mouse fetal ovarian culture in vitro}

Mouse $16.5 \mathrm{dpc}$ fetal ovaries were isolated from pregnant mice, and histological examination of the fetal ovaries revealed that no typical primordial follicles were present (data not shown). Subsequently, each fetal ovary without attachment of mesonephros was cultured in vitro (Fig. 1A). A total of 231 fetal ovaries were cultured in vitro and 197 ovaries (85.3\%) survived after 14 days of culture. Subsequently, the intact early secondary follicles were mechanically isolated from the cultured ovaries using 

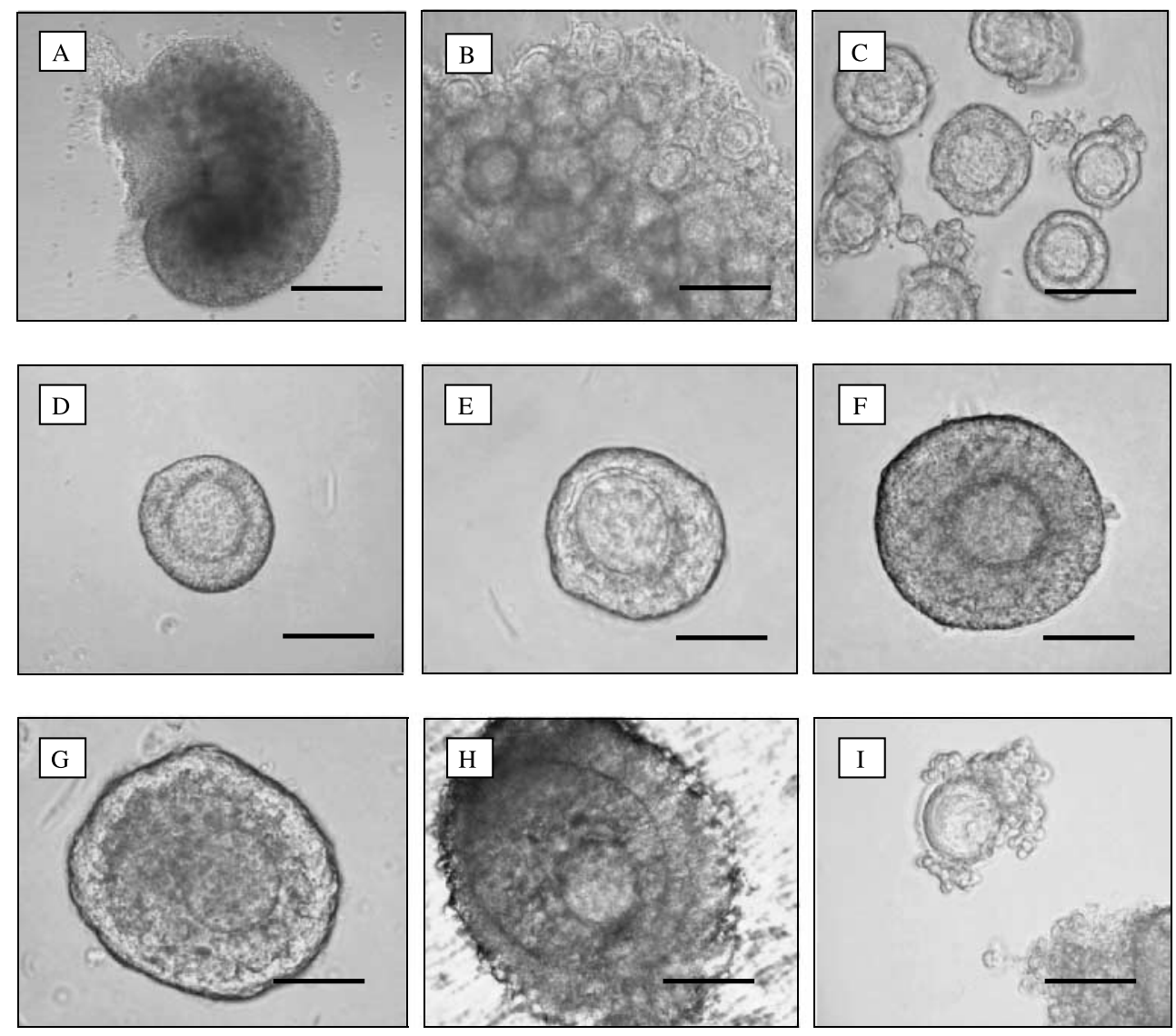

Figure 1 Growth and differentiation characteristics of secondary follicles from fetal ovaries cultured in vitro. (A) shows 16.5 dpc mouse fetal ovary, (B and C) show the fetal ovaries cultured in vitro for 14 days and the secondary follicles isolated from the cultured fetal ovaries respectively. (D-H) show the follicles isolated from ovaries cultured in vitro for 2, 4, 6, 8, and 10 days respectively. (I) shows the oocyte-granulosa cells complexes ovulated from the mature follicle on day 13. Scale bars represent: (A) $500 \mu \mathrm{m}$; (B) $150 \mu \mathrm{m}$; (C, D, and G) $75 \mu \mathrm{m}$; (E and F) $50 \mu \mathrm{m}$; (H and I) $100 \mu \mathrm{m}$.

needles (Fig. 1B and C). Morphological examination of the follicles recovered after 14 days revealed that the follicles had proceeded to the secondary follicle stage. Moreover, the follicles grown in the ovary tissue in vitro were similar to the follicles derived from 12-day-old normal mice. In our experiments, we were able to obtain three to eight intact secondary follicles from a single cultured fetal ovary. We also found that the membrane of secondary follicles derived from mouse ovaries cultured in vitro was able to be breached by needles, and that the granular cells within the follicles were able to be dispersed from the primary oocytes.

\section{Follicular growth characteristics in vitro}

In the experimental group, 678 intact secondary follicles were isolated from 182 mouse ovaries cultured in vitro. These were subsequently cultured in vitro for 12 days. During this period, the follicles progressively grew from a spherical to a diffuse structure and then displayed an antral-like cavity (Fig. 1D-H). A total of 441 follicles $(65.0 \%)$ survived during the 12 -day culture period. From day 8 , an antral-like cavity was observed. A total of 153 follicles that survived (34.7\%) became antral follicles by day 10 , with the COCs located in the antral-like cavity (Fig. $1 \mathrm{H}$ ). In the control group, 314 follicles derived from 12-day-old mouse ovaries were cultured in vitro. Seventy-seven percent of the follicles survived after 12-day culture, and $74 \%$ of the follicles that survived became antral follicles by day 10 in vitro (Shen et al. $2006 a, 2006 b)$. These results indicate that mouse follicular development showed significant differences between the experimental and control groups $(P<0.01)$.

After culturing the cells, we measured the diameters of the oocytes and follicles for a comparison with the experimental and control groups (Fig. 2). In the experimental group, the mean diameter of the oocytes $(n=183)$ was $72.1 \pm 5.4 \mu \mathrm{m}$, while in the control group, it $(n=104)$ was $76.9 \pm 4.1 \mu \mathrm{m}$. The mean diameters of the follicles from the ovaries cultured for 12 days in vitro $(n=158)$ and the control group $(n=114)$ were $347 \pm 53$ and $413 \pm$ $44 \mathrm{~mm}$ respectively. These data regarding oocytes and follicle showed no significant differences between the experimental and control groups. Compared with published results (Liu et al. 2000, 2001, Lenie et al. 2004, Shen et al. 2006a, 2006b), the sizes of the oocytes and follicles 


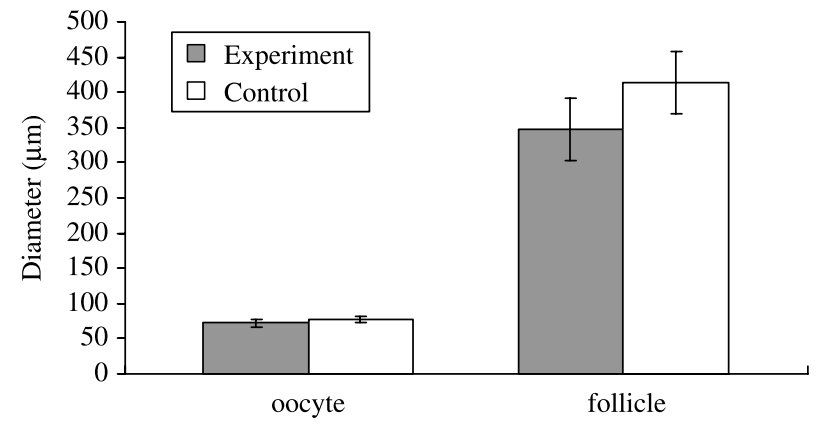

Figure 2 Characteristics of mouse follicles and oocytes derived from a $16.5 \mathrm{dpc}$ fetal ovary in vitro. Diameters of the oocytes and the follicles in the experimental and control groups grown in vitro respectively.

generated from the fetal germ cells in this study were similar to that from the secondary follicles cultured in vitro.

In the experimental group, the concentration of $\mathrm{E}_{2}$ progressively increased as the time of incubation also increased (Fig. 3A). However, it remained lower than that from the control cultures of early preantral follicles. The basal progesterone production before hCG stimulus remained higher in the cultures of experimental group than in the cultures of control group. Subsequently, the production of progesterone increased dramatically at $18 \mathrm{~h}$ after stimulation with hCG/EGF inducing ovulation, and the progesterone production stayed lower in the cultures of experimental group than in the cultures of control group (Fig. 3B). There was no significant difference between the experimental and control groups in the production of $E_{2}$ and progesterone.
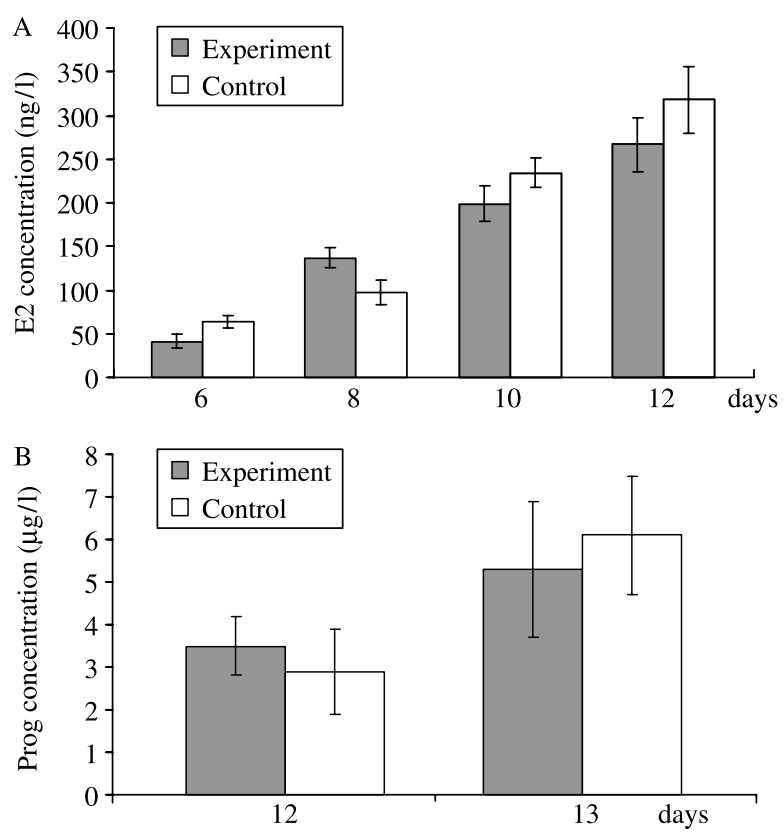

Figure 3 (A) Estradiol concentration (ng/l) in media of the experimental and control groups. (B) Progesterone concentration $(\mu \mathrm{g} / \mathrm{l})$ in conditioned media of the experimental and control groups.

\section{Molecular characterization of oocytes derived from fetal germ cells in vitro}

We further examined the expression of follicle-specific genes (Ddx4, Gdf9, Dmc1h, Og2x, Sycp3, and Lhcgr) in different stages of in vitro-cultured follicles by RT-PCR. As shown in Fig. 4, expression of these follicle-specific genes was detected as early as day 0 in the follicle culture in vitro, which is equal to mouse ovary in vivo around postnatal day 12. In general, their expression patterns were similar to that of the follicles from various stages of postnatal mouse ovaries (Hübner et al. 2003, Lenie et al. 2004, Rajkovic et al. 2004, Shen et al. 2006b).

To determine whether methylation of imprinted genes was completed during the oogenesis observed during our in vitro culture method, we examined the differentially methylated regions (DMRs) of the maternal imprinted gene Igf $2 r$ in the oocytes of the experimental group. In mammals, parental imprinted memories persist in somatic cells after fertilization, and it is necessary for them to be erased and re-established during germ cell development to reflect the gender of the individual. The erasure process of genomic imprinting memory proceeds in mouse primordial germ cells (PGCs) from 10.5 to 11.5 $\mathrm{dpc}$, and the maternal-specific imprints are established during oogenesis (Kaneko-Ishino et al. 1995, Kono et al. 1996, Miyoshi et al. 1998, Obata et al. 1998, 2002 b, Bao et al. 2000, Surani 2001, Lee et al. 2002, Zwaka \& Thomson 2005). The Igf $2 r$ gene, a maternally expressed imprinted gene, has been shown with a fully methylated pattern in the maternal allele and an unmethylated pattern in the paternal allele in somatic cells, and begins to be methylated in mouse oocytes after $13.5 \mathrm{dpc}$ (Brandeis et al. 1993). As shown in Fig. 5, we analyzed the DNA methylation status in DMRs of Igf2 $r$ and in the control gene peg3, a paternally expressed imprinted gene. In experimental group, the Igf $2 r$ gene was methylated at DMR CpG sites in the oocytes developed

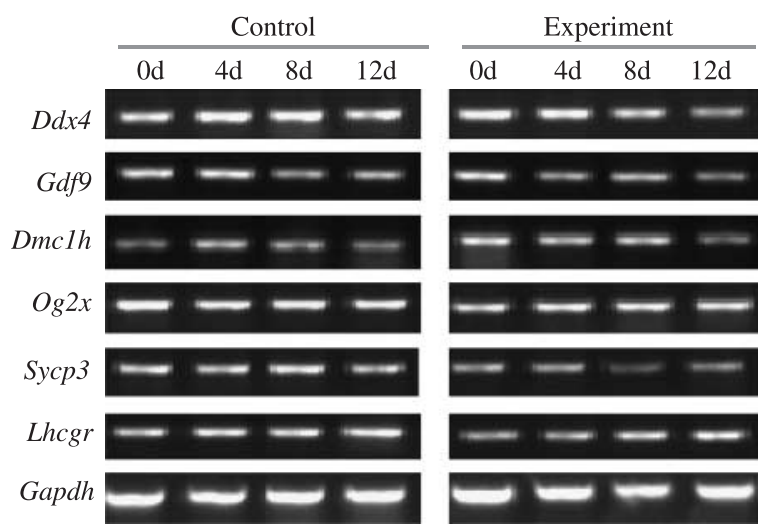

Figure 4 Expression of follicle stage-specific genes in mouse follicles derived from $16.5 \mathrm{dpc}$ fetal ovary after in vitro tissue culturing for 14 days. Detection of follicle-specific gene mRNA in different stages including $0,4,8$, and 12 days in vitro grown follicles from the fetal ovaries in the experimental and control groups. 
in vitro, and the DNA clones with methylated sites accounted for more than $65.5 \%$ of all the CpG sites. The results of the methylated patterns of imprinted gene $\lg f 2 r$ show the significant difference among the experimental group, the control group (92.2\%), and the normal oocytes $(96.3 \% ; P<0.01)$.

\section{Oocyte maturation and fertilization in vitro}

To test the developmental capacity of the oocytes derived from fetal germ cells in vitro, COCs from the inside of the antral follicles were matured in a medium supplemented with hCG and EGF for 16-18 h. The results are summarized in Tables 1 and 2. The oocytes which extruded the first polar body were judged to be metaphase II.

In the experimental group, 141 oocytes inside antral follicles were matured in vitro, 102 (72.3\%) oocytes underwent GVBD and progressed to MII stage achieving $53.9 \%$ of GVBD oocytes (Fig. 6A; Table 1). In the control group, 139 oocytes were matured in vitro, $79.9 \%$ oocytes underwent GVBD and progressed to MII stage achieving $57.7 \%$ of GVBD oocytes. There were no differences in the GVBD formation rates and MII oocyte rates, when comparing the experimental and control groups.

To assess the capacity of the matured oocytes derived from the fetal germ cells cultured in vitro, GVBD oocytes from the experimental and control groups were fertilized in vitro, and zygotes were examined for cleavage to the two-cell stage $24 \mathrm{~h}$ after fertilization in vitro and for late morula and early blastocyst developmental competence in vitro. In the experimental group, 97 oocytes were fertilized, 44 embryos (45.4\%) were able to cleave to the two-cell stage and 15 two-cell stage embryos (34.1\%) could form late morula-early blastocysts (Fig. 6B and C; Table 2). In the control group, 109 oocytes were fertilized, 76 embryos $(69.7 \%)$ could cleave to two-cell stage, and 53 two-cell stage embryos (69.7\%) could form the late morula or early blastocysts. There was a significant difference in the two-cell embryonic $(P<0.05)$ and blastocyst development rates $(P<0.01)$ between the experimental and control groups.

\section{Discussion}

In this study, we obtained mature oocytes from $16.5 \mathrm{dpc}$ mouse fetal germ cells in vitro. Our results demonstrate that $16.5 \mathrm{dpc}$ mouse fetal germ cells are able to form primordial follicles with pregranulosa cells in vitro, and that the oocytes within the growing follicles are able to mature and support the embryonic preimplantation development in vitro. Furthermore, we confirmed that the erasure of genomic imprinting markers that had been imposed in the parental generation was imposed in the oocytes that developed from fetal germ cells.

Before the formation of the primordial follicles, germ cell clusters undergo programmed breakdown and massive apoptosis of oocytes during the later stages of
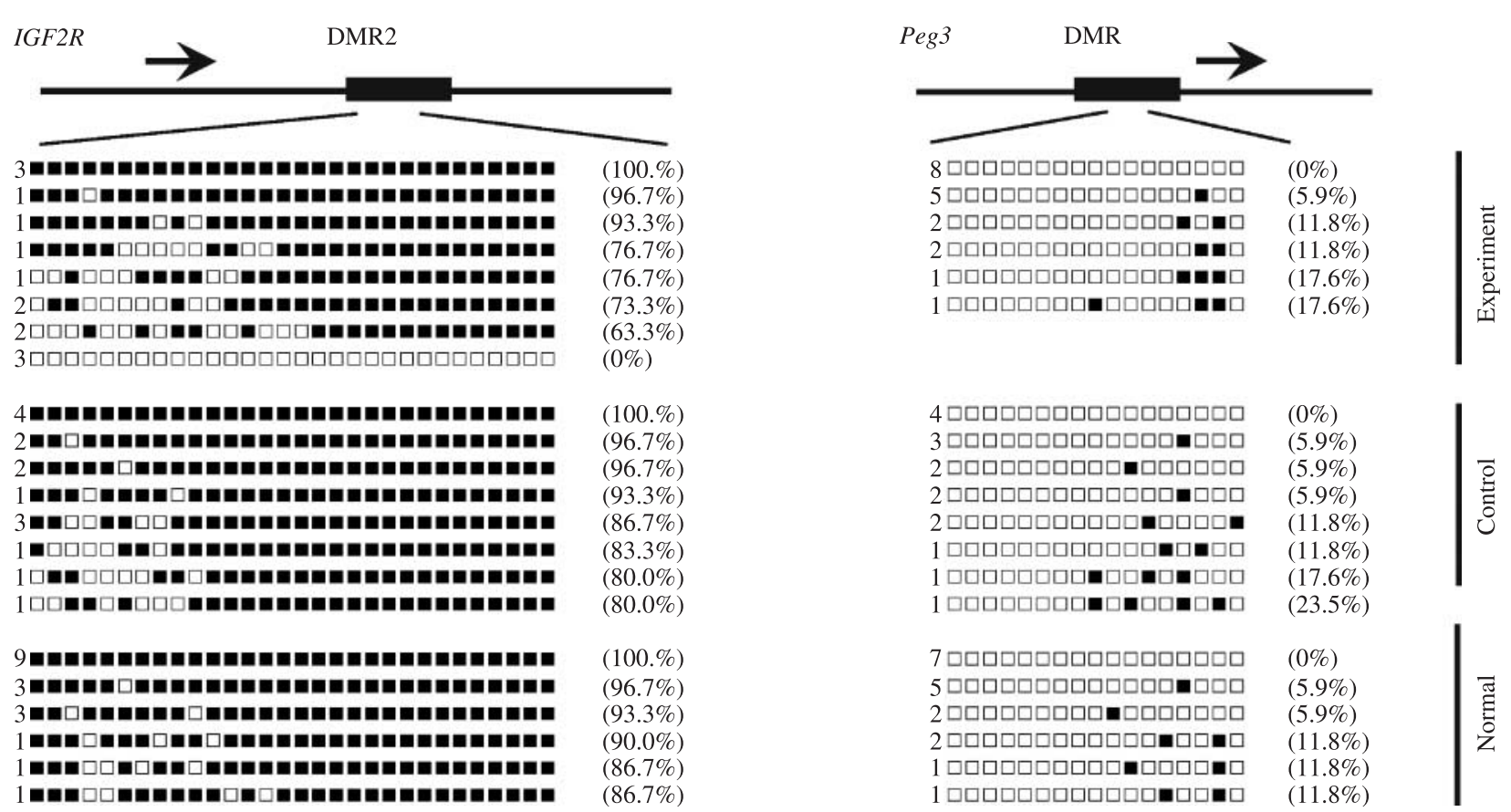

Figure 5 Methylation status of the differentially methylated regions (DMRs) of the imprinted genes, lgf $2 r$ and peg3, in the oocytes from experimental group, control group, and normal mice. Each line represents an individual clone. In total, $30 \mathrm{CpG}$ sites within the DMR of Igf2r gene, and $17 \mathrm{CpG}$ sites within the DMR of peg3 gene were analyzed. Squares indicate CpG sites within the regions analyzed; filled and open squares indicate methylated and unmethylated cytosines respectively. The number of DNA samples sequenced (left) and the percentage of the methylated CpG sites out of all the CpG sites (right) are represented to the left and right of each line respectively. 
Table 1 In vitro maturation of mouse oocytes derived from fetal germ cells.

\begin{tabular}{lccc}
\hline Groups & No. of oocytes & $\begin{array}{c}\text { No. of GVBD } \\
\text { oocytes }\end{array}$ & $\begin{array}{c}\text { No. of MII } \\
\text { oocytes }\end{array}$ \\
\hline $\begin{array}{l}\text { Experimental } \\
\text { group }\end{array}$ & 141 & $102(72.3 \%)$ & $55(39.0 \%)$ \\
\begin{tabular}{l} 
Control group \\
\hline
\end{tabular} & 139 & $111(79.9 \%)$ & $64(46.0 \%)^{*}$ \\
\hline
\end{tabular}

A total of 141 oocytes were matured in vitro and the ratios of germinal vesicle breakdown (GVBD) and MII oocytes are in terms of the GVBD and MII oocytes from the GV oocytes respectively. ${ }^{*} P<0.05$.

gestation. Only $33 \%$ of oocytes were able to survive and were surrounded by a single layer of granulosa cells to form the primordial follicles (Pepling \& Spradling 1998, 2001). These problems caused a decrease in the rate of formation and development of the primordial follicles. Researchers found that mouse oocytes derived from fetal germ cells were unable to resume meiosis and progress to the last stage of growth in vitro (Klinger \& de Felici 2002, Obata et al. 2002a, Niwa et al. 2004, Farini et al. 2005). The efforts to solve these problems with an in vitro culture system were limited because very little is known about the mechanisms underlying the initiation of oocyte growth, the molecular interactions between the oocytes and somatic cells during the follicle formation, the formation of gap junctions in the follicle, and the growth initiation and selective mechanisms of the follicle (Klinger \& de Felici 2002, Lee et al. 2002).

Several possible factors might account for the incompetence of oogenesis in vitro. One is the abnormal gap junction between the follicular granulosa cells and oocytes that might influence the development of oocytes and block the meiosis (Carabatsos et al. 2000, Eppig 2001, Klinger \& de Felici 2002, Hahn et al. 2005). Several studies have demonstrated that the gap junctions during the development of follicles were required for the progression of the follicular development, and the intercellular connections were thought to be important for transporting small molecules facilitating the growth of mouse oocyte (Carabatsos et al. 2000, Eppig 2001, Epifano \& Dean 2002, Matzuk et al. 2002). Klinger et al. demonstrated that mouse oocytes cultured in vitro for 18-20 days derived from 15.5 to $16.5 \mathrm{dpc}$ fetal germ cells were unable to complete the final growth stage

Table 2 In vitro fertilization of mouse oocytes derived from fetal germ cells.

\begin{tabular}{lccc}
\hline Groups & $\begin{array}{c}\text { No. of } \\
\text { fertilized } \\
\text { oocytes }\end{array}$ & $\begin{array}{c}\text { No. of two-cell } \\
\text { embryos }\end{array}$ & $\begin{array}{c}\text { No. of morula/ } \\
\text { blastocysts }\end{array}$ \\
\hline $\begin{array}{l}\text { Experimental } \\
\text { group }\end{array}$ & 97 & $44(45.4 \%)$ & $15(15.5 \%)$ \\
Control group & 109 & $76(69.7 \%)^{*}$ & $53(48.6 \%)^{+}$ \\
\hline
\end{tabular}

Ninety-seven GVBD oocytes were fertilized in vitro and the ratio of developmental embryo is in terms of the embryos from the GVBD oocytes. ${ }^{*} P<0.05,{ }^{\dagger} P<0.01$. corresponding to the in vivo antral follicle stage, and insufficient maintenance of gap junction communication between follicular oocyte and granulosa cells in vitro reduced paracrine signaling transduction and blocked the oocyte growth and development (Klinger \& de Felici 2002). This in turn could depend on accompanying granulosa cell differentiation regulated by gonadotropins and by signals from the oocyte itself (Eppig \& O'Brien 1996, Eppig et al. 1996). In this regard, selective deletion of connexins expressed in the follicle has resulted that defects both in signaling between oocyte and granulosa cells and intercellular coupling between granulosa cells severely affected oocyte growth and the acquisition of meiotic competence. In fact, oocytes lacking connexin37 are able to grow up to a maximum diameter of about $50 \mu \mathrm{m}$ (Simon et al. 1997), similar to that achieved by the oocytes in Klinger's culture system (Klinger \& de Felici 2002). Similarly, oocytes from connexin-43-deficient mice can grow at decreased rate (final mean diameter $53 \mu \mathrm{m}$ ) and fail to undergo GVBD (Ackert et al. 2001). Another cause is absence of synchrony between nuclear and cytoplasm maturation, which is necessary for the acquisition of the competence to resume meiosis (McLaren \& Buehr 1990, McLaren \& Southee 1997 Chuma \& Nakatsuji 2001, Pepling \& Spradling 2001, Obata et al. 2002a). In order to resume meiosis, oocyte volume needs to reach $80 \%$ of its maximal volume; mouse oocytes generated in vitro are unable to reach this size (Eppig \& O'Brien 1996, Eppig et al. 1996). The premeiotic germ cells within mouse fetal ovary are able to be induced into GV stage oocytes in vitro. However, these GV stage oocytes could not mature in vitro due to their incompetent cytoplasm (Bao et al. 2000, Obata et al. 2002a, Niwa et al. 2004). Recently, incompetence of folliculogenesis in vitro was solved using a two-step culture method utilizing a combination of in vivo transplantation under the kidney capsule and in vitro culture (Shen et al. 2006a, 2006b). Our early research demonstrated that the fetal germ cells were able to complete two meiotic divisions and generate a large number of mature oocytes, which were highly competent in supporting the embryonic development in vitro (Shen et al. 2006a,b). Mouse kidney capsule, as an ectopic site, provides a suitable environment for the development of mouse follicles (Cox et al. 1996, 2000, Liu et al. 2000, 2001). However, whether mouse oocytes derived from the fetal germ cells without the environment of kidney capsule were able to resume meiosis and progress to the last stage of growth in vitro is still unknown, and advanced research should be done for the critical factors required for the induction of meiosis in vitro.

In previous studies, it was reported by Eppig et al. that the oocyte-granulosa cell complexes from mice younger than 6 days of age are unable to sustain development in vitro (Eppig \& O’Brien 1996, Eppig et al. 1996). However, early secondary mouse follicles from 8- or 12-day-old mouse ovaries could be cultured and 

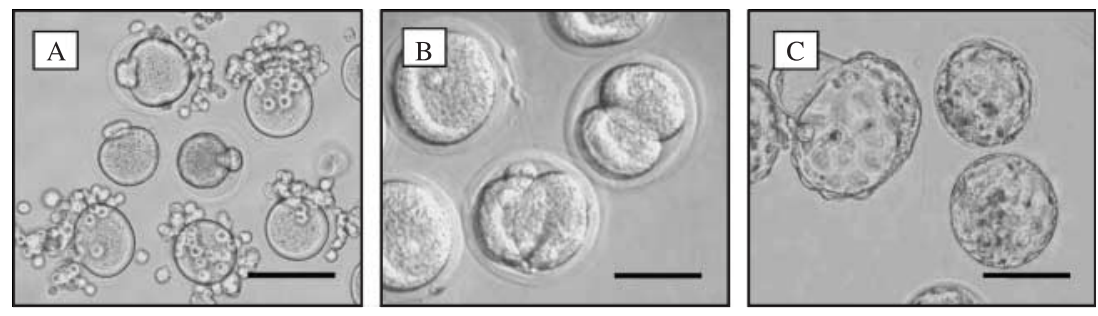

Figure 6 In vitro maturation and fertilization of oocytes from cultured fetal ovaries in vitro. (A) The oocytes matured in vitro derived from $16.5 \mathrm{dpc}$ mouse fetal ovaries. (B and C) Development of oocytes derived from in vitro culturing after fertilization (A). The bars of A, B, and C represent 100,50 , and $100 \mu \mathrm{m}$ respectively.

matured in vitro (Liu et al. 2001, Lenie et al. 2004). When the ovaries of newborn mice were cultured in vitro with whole organ for 8 days, and then the growing oocyte-granulosa cell complexes were isolated from the organ-cultured ovaries and cultured for an additional 14 days, these oocytes were able to be fertilized in vitro, and supported the development to term (Eppig \& O'Brien 1996, Eppig et al. 1996, O'Brien et al. 2003). The culture conditions for purified mouse PGCs in the absence of somatic cell support had been established to study the molecular dissection of the processes governing the development of such cells crucial for early gametogenesis (Farini et al. 2005). Under these culture conditions, PGCs were able to proliferate at high rate, undergo meiosis, and even to progress to a completed meiotic prophase I; however, they did not go on to grow and enter the meiotic prophase II. However, PGCs, when cultured within intact mouse fetal ovary, are able to be induced into GV stage oocytes in vitro and to mature in culture by transferring their nuclei into enucleated oocytes fully grown in vivo (Obata et al. 2002a, Niwa et al. 2004).

It seems that the ovarian environment is crucial for the early development of mouse follicles before postnatal day 8. Eppig (2001) and Epifano \& Dean (2002) further demonstrated that the communication between oocytes and granulosa cells is bidirectional, and a complex interaction of regulatory factors regulates the development of both types of cells, which is essential not only for oocyte development but also for follicular development beginning with the initial assembly of the primordial follicle and continuing throughout ovulation. In our initial experiments, germ cells were isolated from $16.5 \mathrm{dpc}$ mouse fetal ovaries and aggregated into ovary-like cell clusters with the $16.5 \mathrm{dpc}$ fetal ovarian somatic cells. Furthermore, by in vitro culturing, only a few primordial follicles can be formed in the aggregated cell clusters (data not shown). Therefore, we changed our strategy and used fetal germ cells preserved in the natural fetal ovary as the starting materials, rather than isolated primary oocytes, and then performed tissue culture in vitro for 14 days (including 2 days fetal ovarian development and 12 days postnatal ovarian development). Subsequently, the early secondary follicles of the fetal ovary were isolated as soon as possible and cultured in vitro for 12 days, following the method of previous studies (Eppig \& O'Brien 1996, Eppig et al. 1996, O'Brien et al. 2003, Lenie et al. 2004, Liu et al. 2000, 2001,
Shen et al. 2006a, 2006b). Our results demonstrated that incompetent folliculogenesis in vitro can be overcome by this two-step method utilizing a combination of 14-day ovarian tissue culture in vitro and 12-day single follicle culture in vitro, and further suggested that the non-serum medium is able to promote the formation of primordial follicles in our experiment other than the serum medium described in O'Brien experiment, and somatic cell support in the context of the intact $16.5 \mathrm{dpc}$ mouse fetal ovary is most favorable for the development of germ cells in vitro. Furthermore, in our experiments, mouse fetal ovaries were cultured in vitro before follicular formation as described by Eppig's group (Eppig \& O'Brien 1996, Eppig et al. 1996, O'Brien et al. 2003), Together, the detailed mechanism of interaction between mouse somatic and germ cells to form the follicles and their initiation into a growth phase in vitro would enable us to exploit the primordial follicle as a starting point for regulating mammalian fertility (Eppig 2001, Epifano \& Dean 2002). This study may be valuable for mouse folliculogenesis. In future, we will further optimize the method and attempt to accomplish the whole process of mouse oogenesis in vitro from mouse PGC.

\section{Acknowledgements}

This work is supported by the Key Fund for Doctoral Scientific Research Startup of Qingdao Agricultural University (630615) and the Ministry of Science and Technology Grant (2007CB947401) to W Shen, the Key Programs for Science and Technology Development of Shandong province (J05K06) to Q J Pan, and Bill \& Melinda Gates Foundation Grant (37871) to $\mathrm{H}$ Deng. The authors declare that there is no conflict of interest that would prejudice the impartiality of this scientific work.

\section{References}

Ackert CL, Gittens JEI, O'Brien MJ, Eppig JJ \& Kidder GM 2001 Intercellular communication via connexin 43 gap junctions is required for ovarian folliculogenesis in the mouse. Developmental Biology $233258-270$.

Bao S, Obata Y, Carroll J, Domeki I \& Kono T 2000 Epigenetic modifications necessary for normal development are established during oocyte growth in mice. Biology of Reproduction 62 616-621.

Brandeis M, Kafri T, Ariel M, Chaillet JR, McCarrey J, Razin A \& Cedar H 1993 The ontogeny of allele-specific methylation associated with imprinted genes in the mouse. EMBO Journal 12 3669-3677. 
Byskov AG, Xia G \& Andersen CY 1997 The cortex-medulla oocyte growth pattern is organized during fetal life: an in vitro study of the mouse ovary. Molecular Human Reproduction 3 795-800.

Carabatsos MJ, Sellitto C, Goodenough DA \& Albertini D 2000 Oocyte-granulosa cell heterologous gap junctions are required for the coordination of nuclear and cytoplasmic meiotic competence. Developmental Biology 226 167-179.

Chuma S \& Nakatsuji N 2001 Autonomous transition into meiosis of mouse fetal germ cells in vitro and its inhibition by gp130-mediated signaling. Developmental Biology 229 468-479.

Cox SL, Shaw J \& Jenkin G 1996 Transplantation of cryopreserved fetal ovarian tissue to adult recipients in mice. Journal of Reproduction and Fertility 107 315-322.

Cox SL, Shaw J \& Jenkin G 2000 Follicular development in transplanted fetal and neonatal mouse ovaries is influenced by the gonadal status of the adult recipient. Fertility and Sterility 74 366-371.

Epifano O \& Dean J 2002 Genetic control of early folliculogenesis in mice. Trends in Endocrinology and Metabolism 13 169-173.

Eppig JJ 2001 Oocyte control of ovarian follicular development and function in mammals. Reproduction 122 829-838.

Eppig JJ \& O'Brien MJ 1996 Development in vitro of mouse oocytes from primordial follicles. Biology of Reproduction 54 197-207.

Eppig JJ, O'Brien MJ \& Wigglesworth K 1996 Mammalian oocytes growth and development in vitro. Molecular Reproduction and Ddevelopment 44 260-273.

Farini D, Scaldaferri ML, Iona S, Sala GL \& de Felici M 2005 Growth factors sustain primordial germ cell survival, proliferation and entering into meiosis in the absence of somatic cells. Developmental Biology 285 49-56.

Hahn KL, Johnson J, Beres BJ, Howard S \& Wilson-Rawls J 2005 Lunatic fringe null female mice are infertile due to defects in meiotic maturation. Development 132 817-828.

Hübner K, Fuhrmann G, Christenson LK, Kehler J, Reinbold R, Fuente RDL, Wood J, Strauss JF, Boiani M \& Scholer HR 2003 Derivation of oocytes from mouse embryonic stem cells. Science 300 1251-1256.

Kaneko-Ishino T, Kuroiwa Y, Miyoshi N, Kohda T, Suzuki R, Yokoyama M, Viville S, Barton SC, Ishino F \& Surani MA 1995 Peg1/Mest imprinted gene on chromosome 6 identified by cDNA subtraction hybridization. Nature Genetics 11 52-59.

Kezele PR, Ague JM, Nilsson E \& Skinner MK 2005 Alterations in the ovarian transcriptome during primordial follicle assembly and development. Biology of Reproduction 72 241-255.

Klinger FG \& de Felici M 2002 In vitro development of growing oocytes from fetal mouse oocytes: stage-specific regulation by stem cell factor and granulosa cells. Developmental Biology 244 85-95.

Kono T, Obata Y, Yoshimzu T, Nakahara T \& Carroll J 1996 Epigenetic modifications during oocyte growth correlates with extended parthenogenetic development in the mouse. Nature Genetics 13 91-94.

Lee J, Inoue K, Ono R, Ogonuki N, Kohda T, Kaneko-Ishino T, Ogura A \& Ishino F 2002 Erasing genomic imprinting memory in mouse clone embryos produced from day 11.5 primordial germ cells. Development 129 1807-1817.

Lenie S, Cortvrindt R, Adriaenssens T \& Smitz J 2004 A reproducible two-step system for isolated primary mouse ovarian follicles as single functional units. Biology of Reproduction 71 1730-1738.

Liu J, der Elst JV, den Broecke RV, Dumortier F \& Dhont M 2000 Maturation of mouse primordial follicles by combination of grafting and in vitro culture. Biology of Reproduction 62 1218-1223.

Liu J, der Elst JV, den Broecke RV \& Dhont M 2001 Live offspring by in vitro fertilization of oocytes from cryopreserved primordial mouse follicles after sequential in vivo transplantation and in vitro maturation. Biology of Reproduction 64 171-178.
Matzuk MM, Burns KH, Viveiros MM \& Eppig JJ 2002 Intercellular communication in the mammalian ovary: oocytes carry the conversation. Science 296 2178-2180.

McLaren A 2000 Germ and somatic cell lineages in the developing gonad. Molecular and Cellular Endocrinology 163 3-9.

McLaren A \& Buehr M 1990 Development of mouse germ cells in cultures of fetal gonads. Cell Differentiation and Development 31 185-195.

McLaren A \& Southee D 1997 Entry of mouse embryonic germ cells into meiosis. Developmental Biology 187 107-113.

Miyoshi N, Kuroiwa Y, Kohda T, Shitara H, Yonekawa H, Kawabe T, Hasegawa H, Barton SC, Surani MA, Kaneko-Ishino T et al. 1998 Identification of the Meg1/Grb10 imprinted gene on mouse proximal chromosome 11, a candidate for the silver-russell syndrome gene. PNAS 95 1102-1107.

Niwa K, Takano R, Obata Y, Hiura H, Komiyama J, Ogawa H \& Kono T 2004 Nuclei of oocytes derived from mouse parthenogenetic embryos are competent to support development to term. Biology of Reproduction 71 1560-1567.

Obata Y, Kaneko-Ishino T, Koide T, Takai Y, Ueda T, Domeki I, Shiroishi T, Ishino F \& Kono T 1998 Disruption of primary imprinting during oocyte growth leads to the modified expression of imprinted genes during embryogenesis. Development 125 1553-1560.

Obata Y, Kono T \& Hatada I 2002a Maturation of mouse fetal germ cells in vitro. Nature 418 497-498.

Obata Y, Kono T \& Hatada I 2002b Maternal primary imprinting is established at a specific time for each gene throughout oocyte growth. Journal of Biological Chemistry 277 5285-5289.

O'Brien MJ, Pendola JK \& Eppig JJ 2003 A revised protocol for in vitro development of mouse oocytes from primordial follicles dramatically improves their developmental competence. Biology of Reproduction 68 1682-1686.

Pepling ME \& Spradling AC 1998 Female mouse germ cells form synchronously dividing cysts. Development 125 3323-3328.

Pepling ME \& Spradling AC 2001 Mouse ovarian germ cell cysts undergo programmed breakdown to form primordial follicles. Developmental Biology 234 339-351.

Rajkovic A, Pangas SA, Ballow D, Suzumori N \& Matzuk MM 2004 Nobox deficiency disrupts early folliculogenesis and oocyte specific gene expression. Science 305 1157-1159.

Shen W, Li L, Zhang DH, Pan QJ, Ding MX \& Deng HK 2006a Mouse oocytes derived from fetal germ cells are competent to support the development of embryos by in vitro fertilization. Molecular Reproduction and Ddevelopment 73 1312-1317.

Shen W, Zhang DH, Qing TT, Cheng J, Bai ZD, Shi YQ, Ding MX \& Deng HK $2006 b$ Living offspring produced by mouse oocytes derived from premeiotic fetal germ cells. Biology of Reproduction 75 615-623.

Simon AM, Goodenough DA, Li E \& Paul DL 1997 Female infertility in mice lacking connexin 37. Nature 385 525-529.

Smitz JEJ \& Cortvrindt RG 2002 The earliest stages of folliculogenesis in vitro. Reproduction 123 185-202.

Surani MA 2001 Reprogramming of genome function through epigenetic inheritance. Nature 414 122-128.

Zwaka TP \& Thomson JA 2005 A germ cell origin of embryonic stem cells? Development 132 227-233.

Received 18 December 2006

First decision 27 February 2007

Revised manuscript received 26 April 2007

Accepted 9 May 2007 